\section{§30. Quantum Oscillations of Thermoelectric Effects in a Pseudo-one-dimensional Electron Gas with a Spin-orbit Interaction}

Nakamura, H., Hatano, N., Hirayama, N. (Univ. Tokyo), Shirasaki, R. (Yokohama Nat'l Univ.), Yonemitsu, K. (IMS)

We considered thermoelectric effects in a pseudoone-dimensional electron gas (P1DEG) with a spin-orbit interaction $(\mathrm{SOI})^{1}$. This work was supported by the $\mathrm{Na}$ tional Institutes of Natural Sciences (NINS) program for cross-disciplinary study (NIFS10KEIN0160),

In semiconductor spintronics, the electron's spin degree of freedom rather than its electric charge plays the main role in transport, processing and storage of information. When a temperature gradient is applied to a metallic magnet, different driving power should be generated to electrons in different spin channels along the temperature gradient, which is called the spin Seebeck effect ${ }^{2,3)}$. Recently, the spin Seebeck effect was observed in a soft ferromagnetic film $\mathrm{Ni}_{89} \mathrm{Fe}_{19}{ }^{4)}$.

Spins in semiconductor nanostructures can be manipulated by spin-orbit interactions (SOI). The SOI is proportional to $(\boldsymbol{E} \times \boldsymbol{p}) \cdot \boldsymbol{S}$, where $\boldsymbol{E}$ is the electric field, $\boldsymbol{p}$ the momentum and $\boldsymbol{S}$ the spin of the electron. As typical SOIs in III-V semiconductor heterostructures, the Rashba SOI (RSOI) and the Dresselhaus SOI (DSOI) are often discussed. In the RSOI, $\boldsymbol{E}$ is due to structural inversion asymmetry ${ }^{5)}$. On the other hand, $\boldsymbol{E}$ in the DSOI is due to bulk inversion asymmetry ${ }^{6}$.

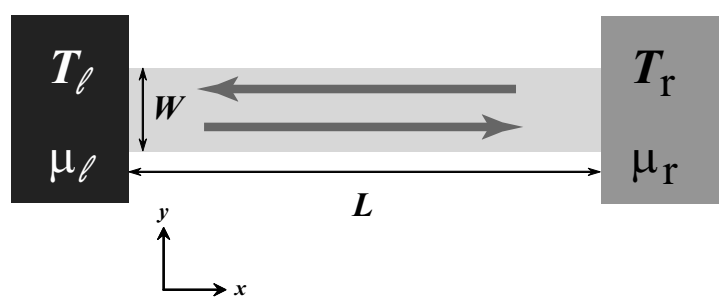

Fig. 1: A quantum wire under electrochemical potential and temperature gradients. The temperature and the electrochemical potential of the left particle bath are $T_{\ell}$ and $\mu_{\ell}$, respectively. Those of the right particle bath are $T_{\mathrm{r}}$ and $\mu_{\mathrm{r}}$. We set $T_{\ell}=T-\Delta T / 2, T_{\mathrm{r}}=T+\Delta T / 2$, $\mu_{\ell}=\mu-\Delta \mu / 2$ and $\mu_{\mathrm{r}}=\mu+\Delta \mu / 2$ with $\Delta T \ll T$ and $\Delta \mu \ll \mu$ for calculating the linear response.

In the present study, we considered thermoelectric effects in the P1DEG system with the RSOI in no magnetic field (Fig. 1). In the thermoelectric effect, an electrochemical potential gradient or a temperature gradient applied to the P1DEG induces an electric current and a heat current. We found by means of theoretical calculation that the transport coefficients of the P1DEG with the RSOI oscillates as the electrochemical potential is changed. The quantum oscillations are due to the van Hove singularities in the density of states ${ }^{7,8)}$.

Moreover, we revealed the relation between the peak positions of the oscillations of these transport coefficients and the edges of the band gaps(Fig. 2). We also reported the violation of the Wiedemann-Franz law in P1DEG with RSOI.

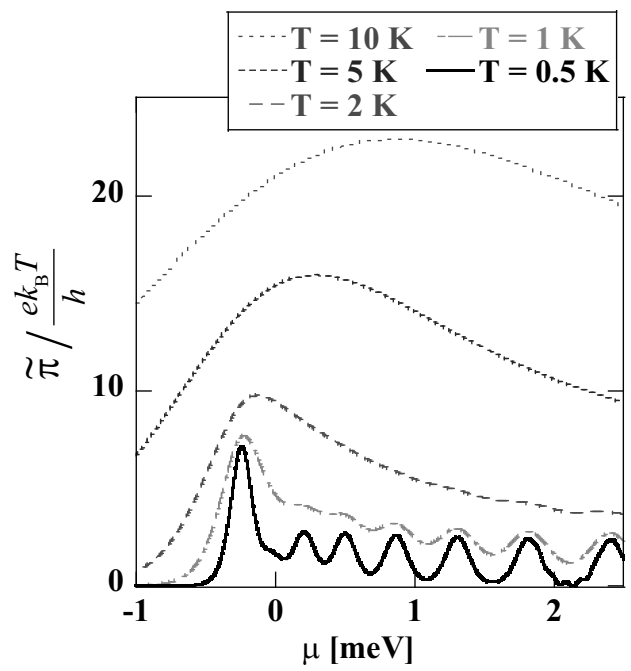

Fig. 2: The transport coefficient against the electrochemical potential $\mu$. This coefficient corresponds to the Seebeck coefficient. As the temperature $T$ decreases, peaks appear.

1) H. Nakamura, N. Hatano, R. Shirasaki, N. Hirayama, K. Yonemitsu, J. Elec. Mat. 40, 601 (2011)

2) L. Gravier, S. Serrano-Guisan, F. Reuse, J.-P. Ansermet, Phys. Rev. B 73, 024419 (2006)

3) O. Tsyplyatyev, O. Kashuba, V.I. Fal'ko, Phys. Rev. B 74, 132403 (2006).

4) K. Uchida, S. Takahashi, K. Harii, J. Jeda, W. Koshibae, K. Ando, S. Maekawa and E. Saitoh, $\mathrm{Na}$ ture 455, 778 (2008).

5) E.I. Rashba, Fiz. Tverd. Tela (Leningrad) 2, 1224 (1960); Sov. Phys. Solid State 2, 1109 (1960).

6) G. Dresselhaus, Phys. Rev. 100, 580 (1955).

7) H. Nakamura, N. Hatano, R. Shirasaki, Solid State Comm. 135, 510 (2005).

8) R. Shirasaki, H. Nakamura, N. Hatano, e-J. Surf. Sci. Nanotech. 3, 518 (2005). 